\section{Endocrine prevention of chemotherapy-induced ovarian failure}

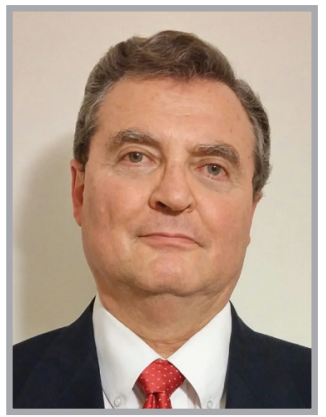

\author{
“Maximizing patients' odds for future fertility \\ necessitates the combination of ovarian \\ cryopreservation, gonadotropin-releasing \\ hormone agonists administration and follicular \\ aspiration. Furthermore, gonadotropin-releasing \\ hormone agonists can effectively prevent the \\ thrombocytopenia-associated menorrhagia in \\ these patients."
}

Zeev Blumenfeld*

First draft submitted: 11 April 2016; Accepted for publication: 12 May 2016; Published online: 2 June 2016

Fertility preservation has gained recent ubiquitous interest. The increase in cancer incidence and the long-term survival improvement have brought about a global augmentation in the struggle for fertility preservation in young women exposed to gonadotoxic chemotherapy $[1-4]$. Among the various methods for fertility preservation, endocrine suppression of ovarian function has been one of the most debatable and equivocal issues [4-13]. However, recent publications moved the pendulum towards acceptance of the beneficial role of gonadotropin-releasing hormone agonists (GnRHa) cotreatment for fertility preservation despite gonadotoxic chemotherapy.

Endocrine ovarian suppression with GnRHa during chemotherapy is an attractive option to preserve gonadal function and fertility with the advantage of causing only minimal delay (1 week) in the initiation of chemotherapy $[4,9]$.

Until a year ago, the scarce data on fertility (pregnancy rate) in the survivors, after endocrine ovarian suppression has been an important limitation, and the American Society of Clinical Oncology (ASCO), the American Society for Reproductive Medicine (ASRM) and the European Society for Medical Oncology (ESMO) did not recommend on this method $[1,4-5,10]$. However, these societies' guidelines are not updated, and new pertinent data have been published $[1,3-4,9]$. Although it is currently considered experimental, recent data suggest both safety and efficacy for the use of GnRHa cotreatment [3-10,12-15].

The philosophy and rationale behind this method is that preventing premature menopause in young female patients is better than treating it after it occurs, according the principle: 'an ounce of prevention is worth a pound of cure' $[1,4]$.

Several possible mechanisms have been put forward to explain the beneficial effect of endocrine ovarian suppression by GnRHa:

- Simulation of a prepubertal, hypogonadotropic milieu;

*Reproductive Endocrinology, RAMBAM Health Care Campus, The Rappaport Faculty of Medicine, Technion - Israel Institute of Technology, Haifa, 31096 Israel; Tel.: +972 4 8542577; Fax: +972 4 8543243;

z_blumenfeld@rambam.health.gov.il

Future
Medicine $\mathrm{fs}$ art of 


\section{"Endocrine ovarian suppression with gonadotropin-releasing hormone agonists during chemotherapy is an attractive option to preserve gonadal function and fertility with the advantage of causing only minimal delay (1 week) in the initiation of chemotherapy."}

- Decreasing ovarian perfusion secondary to the hypoestrogenic milieu;

- Direct effect on ovarian components;

- Intraovarian upregulation of anti-apoptotic molecules such as sphingosine-1-phosphate [S1P]; and

- Possible prevention of gonadotoxicity on oogonial stem cells $[1,4-5]$.

Although the accepted canonical dogma claims the primordial and primary follicles are gonadotropin independent, the more advanced, gonadotropin-dependent follicles, secrete growth factors, such as TGF- $\beta$, bone morphogenic proteins [BMP] 2, 4, 5, 6, 7 and 15, GDF-9, activin and possibly others, which, paracrinically, induce growth of the primordial follicles [5]. The chemotherapy causes follicles atresia, resulting in decreased estrogen and inhibin levels, which feedback to increase follicle-stimulating hormone concentrations, causing the gonadotropin responsive follicles to secrete the above mentioned growth factors which, in a paracrine manner recruit many primordial and primary follicles to enter the unidirectional differentiation and apoptosis (the so called 'burn-out theory') [4]. The chronic effect of GnRHa to decrease follicle-stimulating hormone levels may minimize the activation of primordial follicles and their 'burn-out' [4].

To date, over 22 studies (including eight prospective randomized controlled trials [RCTs]), have reported on 2328 patients treated with GnRHa in parallel to chemotherapy, showing a significant decrease in premature ovarian failure (POF) rate in survivors [4], versus eight studies reporting on 509 patients, where no benefit was demonstrated [4]. Patients treated with GnRHa preserved their cyclic ovarian function $[\mathrm{COF}]$ in almost $90 \%$, as compared with $40-50 \%$ of controls, with pregnancy rates ranging $19-88 \%$ in the GnRHa arm [4]. However, iatrogenic effects may occur with GnRHa administration, such as vaginal dryness, osteopenia, osteoporosis, hot flushes, among others.

Furthermore, 13 recent metaanalyses have concluded that $\mathrm{GnRH}$-a use may be beneficial and decrease the risk of POF in survivors [4-10,12-13].

The only primate prospective RCT with histological assessment of the follicles concentration, examining the follicular loss following exposure to an alkylating agent alone or in parallel to GnRHa has been performed in Rhesus monkeys [14]. The daily follicular decline was
$0.12 \pm 0.012 \%$ for the chemotherapy alone group compared with $0.057 \pm 0.019 \%(\mathrm{p}<0.05)$ for the GnRHa cotreatment. In the cyclophosphamide group $64.6 \pm 2.8 \%$ of the primordial follicle pool were lost compared with only $28.9 \pm 9.1 \%$ in the $\mathrm{GnRHa}+$ cyclophosphamide-treated monkeys $(\mathrm{p}<0.05)$.

Two recent, large, and convincing prospective RCTs were published in the last year $[8,15]$. The POEMS-SWOG S0230 study enrolled only HR-breast cancer patients [15], whereas most patients in the PROMISE-GIM6 [8] study were $\mathrm{HR}+[8,9]$. Both RCTs demonstrated a statistically significant, $70-72 \%$ reduction in ovarian failure rate in the $\mathrm{GnRHa}$ arms (odds ratio [OR]: $0.28-0.30 ; \mathrm{p}<0.001-0.04)[4,8-9,15]$, (OR: $0.30 ; \mathrm{p}=0.04$ ). Moreover, the pregnancy rate was significantly increased by $\mathrm{GnRHa}$ (OR: 2.45; $\mathrm{p}=0.03)[8]$.

Long-term evaluation of the PROMISE-GIM6 study, after a median follow-up of 7.3 years (range: 6.3-8.2 years) [8] has shown a 5-year cumulative menstrual resumption of $72.6 \%$ (95\% CI: 65.7-80.3\%) among the GnRHa group and $64 \%$ among the controls (95\% CI: $1.12-1.95$; $\mathrm{p}=0.006$ ) [9] with no difference in the 5-year disease-free survival (DFS) [8].

In the POEMS-SWOG S0230 study, an NIH-sponsored, prospective RCT trial [15], the GnRHa-treated patients had better-preserved ovarian function across multiple endpoints and improved fertility (more pregnancies) [15]. Unexpectedly, the GnRHa cotreatment led to more favorable DFS and overall survival (OS) rates [15]. Successful pregnancy was achieved by $12 / 18$ women who attempted pregnancy in the chemotherapy alone group compared with $22 / 25$ successful pregnancies in the GnRHa arm (adjusted OR: 2.45; p < 0.03). In addition, women in the GnRHa group gave birth to 18 babies versus 12 in the standard chemotherapy group.

The main drawback of studies evaluating the role of $\mathrm{GnRHa}$ in preserving ovarian function is looking at amenorrhea, lack long-term data of ovarian function and fertility [pregnancies] [3-5]. Therefore, we have recently published the results of pregnancy rate in longterm survivors (up to 25 years) [4]. We have compared 286 patients who received GnRHa + chemotherapy with 188 controls (chemotherapy alone). The OR for preserving COF was 6.87 for the patients who received the GnRHa cotreatment [4]. Furthermore, the total and 
spontaneous pregnancy rate was significantly higher for those who received the GnRHa ( $p$ $<0.006$ ). More survivors in the GnRHa group resumed COF versus controls (87 vs 49\%; OR: 6.87; $\mathrm{p}<0.0001)$ [4]. In total, 90 patients $(61 \%)$ conceived in the GnRHa group versus 31 (42\%) in the control group $(\mathrm{p}<0.0003)$, resulting in 129 and 41 newborns $(\mathrm{p}<0.01)$, respectively [4]. Spontaneous pregnancies occurred in 80 survivors $(58 \%)$ in the $\mathrm{GnRH}$ a group versus $25(35 \%)$ in the controls (OR: 3.12; p < 0.0004) [4].

The achievement of fertility (pregnancies) in our study is in keeping with the two recent large RCTs $[8,15]$. Similarly these three studies have also found significant reduction in POF in the GnRHa arms (OR: 0.28-0.3; $\mathrm{p}<0.001-0.04)[4,8,15]$.

The recent 14th St Gallen international conference and expert consensus [13], also supports the use of GnRHa in breast cancer patients stating that GnRHa therapy during chemotherapy proved effective to protect against POF and preserve fertility. This consensus states that the GnRHa cotreatment also increased the rate of subsequent successful pregnancies and did not compromise disease outcomes [13].

However, not all the studies are supportive of GnRHa cotreatment. In the meta-analysis by Elgindy $e t$ al., the ovarian suppression by GnRHa did not significantly increase the COF $(\mathrm{p}=0.07)$ [11]. This meta-analysis has been criticized of assigning lower weight to the two recent, large, RCT $[8,15]$ and excluding RCTs in support of GnRHa, with a possible consequent underestimate of the beneficial effect of the GnRHa cotreatment. In this connection, the study by Demeestere et al. [16], which has been included in the above problematic meta-analysis [11], has not found, initially, a difference in POF rate after 1 year; However, Demeestere et al. have subsequently presented at the 3rd World Congress of the International Society for Fertility Preservation in Valencia, Spain, November 2013, that at 2 years follow-up of the same patients included in their previous publication [17] declaring that: "...the number of patients who totally restored their ovarian function was significantly higher in the GnRHa group ( $\mathrm{p}=0.049)$ confirming results of significantly higher AMH" levels in the GnRHa arm $(1.4 \mathrm{ng} / \mathrm{ml})$ vs the control arm; $\mathrm{AMH}=0.5 \mathrm{ng} / \mathrm{ml} ; \mathrm{p}=0.04$. Indeed, short follow-up may be responsible for the discrepancy between studies and lead to incorrect conclusions $[1,4-5]$.
Since the ultimate gold standard of fertility preservation is pregnancies, it is notable to mention that high pregnancy rates after $\mathrm{GnRHa}$ cotreatment versus controls was demonstrated in three different studies from three continents:

- In Wong et al. study [18], in the UK, $71 \%$ survivors treated with GnRHa and chemotherapy achieved pregnancy;

- In the POEMS-SWOG study, [15], in the USA, $88 \%$ survivors treated with GnRHa and chemotherapy achieved pregnancy;

- In our study, [3], in Israel $61 \%$ of the survivors spontaneously conceived.

Most relevant to this equivocal and highly debatable issue, a publication [17] from a previous opponent, has found that the use of GnRHa during chemotherapy has also significantly increased the probability to become pregnant (OR: 12.87; $\mathrm{p}=0.001$ ).

Finally, two recent international meetings of experts endorsed the GnRHa beneficial role in fertility preservation $[6,13]$. Ten concluding recommendations were discussed and prepared [6]. The recommendations were graded according to the levels of evidence and grades of recommendation (according to the ESMO Clinical Practice Guidelines for fertility preservation in cancer patients) [6]. The only conclusion (out of the ten) that received the highest grading - I, A, according the above classification was the conclusion regarding GnRHa, namely: "Ovarian suppression with the use of LHRHa during chemotherapy should be considered a reliable strategy to preserve ovarian function and fertility, at least in breast cancer patients, given the availability of new data suggesting both the safety and the efficacy of the procedure have become available (I, A)" [6].

Similarly, the 2015 St Gallen International Expert Consensus panel [13] and the National Comprehensive Cancer Network guidelines [19], noted the value of a GnRHa given during chemotherapy for premenopausal women with ER-negative disease in protecting against POF and preserving fertility.

\section{Conclusion}

Endocrine ovarian suppression with GnRHa during chemotherapy is still considered experimental, although recent data suggest safety and efficacy $[1,3-4,8,13,15]$. Therefore, it should be administered as part of clinical trials with

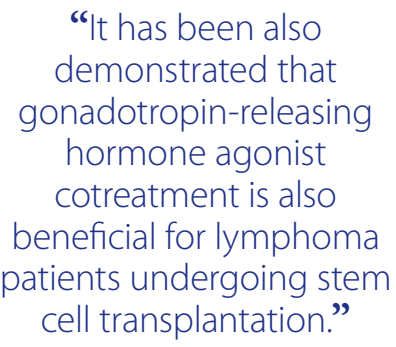

"It has been also

demonstrated that onadotropin-releasing hormone agonist cotreatment is also patients undergoing stem cell transplantation." 
patients signing informed consent. Maximizing patients' odds for future fertility necessitates the combination of ovarian cryopreservation, GnRHa administration and follicular aspiration. Furthermore, GnRHa can effectively prevent the thrombocytopenia-associated menorrhagia in these patients [1,3-4].

It has been also demonstrated that GnRHa cotreatment is also beneficial for lymphoma patients undergoing stem cell transplantation [1,3-4,20-21].
Financial \& competing interests disclosure The author has no relevant affliations or financial involvement with any organization or entity with a financial interest in or financial conflict with the subject matter or materials discussed in the manuscript. This includes employment, consultancies, honoraria, stock ownership or options, expert testimony, grants or patents received or pending, or royalties.

No writing assistance was utilized in the production of this manuscript.

\section{References}

1 Blumenfeld Z, Katz G, Evron A. 'An ounce of prevention is worth a pound of cure': the case for and against GnRH-agonist for fertility preservation. Ann. Oncol. 25(9), 1719-1728 (2014).

2 Kim SY, Kim SK, Lee JR, Woodruff TK. Toward precision medicine for preserving fertility in cancer patients: existing and emerging fertility preservation options for women. J. Gynecol. Oncol. 27, e22 (2016).

3 Blumenfeld Z, Zur H, Dann EJ. Gonadotropin-releasing hormone agonist cotreatment during chemotherapy may increase pregnancy rate in survivors. Oncologist 20 (11), 1283-1289 (2015).

4 Blumenfeld Z, Evron A. Preserving fertility when choosing chemotherapy regimens - the role of gonadotropin-releasing hormone agonists. Expert Opin. Pharmacother. 16(7), 1009-1020 (2015).

5 Lambertini M, Ginsburg ES, Partridge AH. Update on fertility preservation in young women undergoing breast cancer and ovarian cancer therapy. Curr. Opin. Obstet. Gynecol. 27(1), 98-107 (2015).

6 Lambertini M, Del Mastro L, Pescio MC, Andersen CY. Cancer and fertility preservation: international recommendations from an expert meeting. BMC Med. 14, 1 (2016).

7 Del Mastro L, Ceppi M, Poggio F et al. Gonadotropin-releasing hormone analogues for the prevention of chemotherapy-induced premature ovarian failure in cancer women: systematic review and meta-analysis of randomized trials. Cancer Treat. Rev. 40(5), 675-683 (2014).
8 Lambertini M, Boni L, Michelotti A et al. GIM Study Group. Ovarian suppression with triptorelin during adjuvant breast cancer chemotherapy and long-term ovarian function, pregnancies, and disease-free survival: a randomized clinical trial. JAMA 314(24), 2632-2640 (2015).

9 Del Mastro L, Lambertini M. Temporary ovarian suppression with gonadotropinreleasing hormone agonist during chemotherapy for fertility preservation: toward the end of the debate? Oncologist 20(11), 1233-1235 (2015).

10 Munhoz RR, Pereira AA, Sasse AD et al. Gonadotropin-releasing hormone agonists for ovarian function preservation in premenopausal women undergoing chemotherapy for early-stage breast cancer: a systematic review and meta-analysis. JAMA Oncol. 2(1), 65-73 (2016).

11 Elgindy E, Sibai H, Abdelghani A, Mostafa $\mathrm{M}$. Protecting ovaries during chemotherapy through gonad suppression: a systematic review and meta-analysis. Obstet. Gynecol. 126(1), 187-195 (2015).

12 Lambertini M, Poggio F, Levaggi A, Del Mastro L. Protecting ovaries during chemotherapy through gonad suppression: a systematic review and meta-analysis. Obstet. Gynecol. 126(4), 901 (2015).

13 Coates AS, Winer EP, Goldhirsch A et al. Tailoring therapies-improving the management of early breast cancer: St Gallen International Expert Consensus on the Primary Therapy of Early Breast Cancer 2015. Ann. Oncol. 26(8), 1533-1546 (2015).

14 Ataya K, Rao LV, Lawrence E, Kimmel R. Luteinizing hormone-releasing hormone agonist inhibits cyclophosphamide-induced ovarian follicular depletion in rhesus monkeys. Biol. Reprod. 52(2), 365-372 (1995).

15 Moore HCF, Unger JM, Phillips KA et al. Goserelin for ovarian protection during breast-cancer adjuvant chemotherapy. N. Engl. J. Med. 372(10), 923-932 (2015).

16 Demeestere I, Brice P, Peccatori FA et al. Gonadotropin-releasing hormone agonist for the prevention of chemotherapy-induced ovarian failure in patients with lymphoma: 1-year follow-up of a prospective randomized trial. J. Clin. Oncol. 31(7), 903-909 (2013).

17 Behringer K, Thielen I, Mueller $\mathrm{H}$ et al. Fertility and gonadal function in female survivors after treatment of early unfavorable Hodgkin lymphoma (HL) within the German Hodgkin Study Group HD14 trial. Ann. Oncol. 23(7), 1818-1825 (2012).

18 Wong M, O’Neill S, Walsh G, Smith IE. Goserelin with chemotherapy to preserve ovarian function in pre-menopausal women with early breast cancer: menstruation and pregnancy outcomes. Ann. Oncol. 24(1), 133-138 (2013).

19 The National Comprehensive Cancer Network.

www.nccn.org

20 Blumenfeld Z, Zuckerman T. Repeated spontaneous pregnancies and successful deliveries after repeated autologous stem cell transplantation and $\mathrm{GnRH}$-agonist. Oncologist 15(1), 59-60 (2010).

21 Blumenfeld Z, Patel B, Leiba R, Zuckerman T. Gonadotropin Releasing Hormone agonist may minimize premature ovarian failure in young women undergoing autologous stem cell transplantation. Fertil. Steril. 98(5), 1266-1270 (2012). 